\title{
Network Traffic Prediction Based on Feed-forward Neural Network with PLS Pruning Algorithm
}

\author{
LI Zhenxing, MENG Qinghai \\ PLA 91550 Unit 94, Liaoning Dalian, 116023, China \\ lizhenxing@126.com
}

Keywords: Network Traffic Prediction; FNN; PLS; Pruning Algorithm

\begin{abstract}
To improve the prediction accuracy and reduce the computational complexity of network traffic prediction based on feed-forward neural network (FNN), the partial least squares (PLS) pruning algorithm was proposed to optimize the network topology structure. The data of network traffic has the characteristics of burst, nonlinear and time variation, which results in the traditional neural network has the disadvantages of slow convergence rate and easy to fall into local minimum for network traffic prediction. The performance of FNN is closely related to the number of nodes in the hidden layer, which affects the computational complexity, convergence rate and convergence accuracy. The proposed method uses PLS pruning algorithm to simply the network topology structure, which can obtain the ideal number of hidden layer nodes of the FNN, and then the prediction accuracy of network traffic is improved. The computer simulation results show that the proposed method has faster convergence rate and higher convergence accuracy compared with traditional FNN.
\end{abstract}

\section{Introduction}

With the rapid development of computer technology and network technology, the network scale becomes larger and larger, and internet service has become an indispensable part of people's life [1]. However, the network encountered more and more various technical problems during the process of the network development, such as robustness of network architecture, information security, virus threats and so on. Network traffic prediction can predict the network behavior, analyze the network security situation and guide the network control, which has become a key issue in network management research [2]. Recently there are many reports about the linear and nonlinear methods of network traffic prediction. The linear prediction model mainly includes auto regressive moving average (ARMA) and auto integrated moving average (ARIMA) [3], and the nonlinear prediction model mainly includes neural network prediction model, support machine prediction model and wavelet prediction model. Among the nonlinear prediction models, the neural network model has the ability of nonlinear mapping and good generalization, which has unique advantages in network traffic prediction [4]. In various neural network models, the FNN with error back propagation (BP) algorithm is simple in structure and complete in mathematical theory [5]. BP algorithm is essentially a stochastic gradient descent algorithm, and its convergence rate is slow, and easily falls into the local minimum in the high dimensional optimization problem. When using FNN to solve the nonlinear problem, the network initialization parameters and the network structure have an important influence on the performance. In particular, the neural network topology structure directly affects the computational complexity, convergence rate and convergence accuracy of FNN. There is no theoretical basis for the parameters reasonable initialization and the choice of the neural network topology structure until now. Network traffic prediction predict the future network traffic for a period of time based on the historical network traffic data, which establish the certain relationship between the network traffic history data and the future network traffic data. A large number of research results show that the network traffic time relationship is a complex nonlinear relationship. In order to realize the fast and accurate prediction of network traffic, the neural network optimized by certain algorithm is needed. PLS pruning algorithm is an effective method to optimize the neural network topology structure by using the training data, which can obtain an ideal neural network 
topology structure during the training process. The computer simulation results show that the proposed method has faster convergence rate and lower prediction error compared with traditional FNN neural network with BP algorithm.

\section{Principle of Network Traffic Prediction}

Network traffic is the sum of all the communication in the network link in the unit time. The Network traffic data are usually sampled at the same time during the process of network traffic data collection. So that the time series network traffic data can be obtained. For large-scale nonlinear network system, due to network traffic users are often affected by the outside world has contingency and regularity. Therefore, the corresponding network traffic also has the characteristics of contingency, regularity and unexpected [6]. The network traffic at time $t_{\text {can }}$ be expressed as follow

$$
f(t)=x(t)+\sigma e(t)
$$

Where $x(t)$ is the real network traffic and $e(t)$ is the noise which meets Gaussian distribution, $\sigma$ is the noise variance. Network traffic prediction is to predict the future development trend of network traffic according to the detected historical data of the network traffic, which can provide the basis for network planning and decision-making. The network traffic prediction model can be given by

$$
\hat{y}=f\left(x_{1}, x_{2}, \mathrm{~L}, x_{i}, \mathrm{~L}\right)
$$

Where $\hat{y}$ is the prediction value of the network traffic. The principle of network traffic prediction is shown as Fig.1.

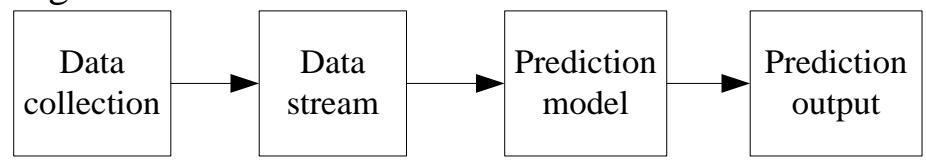

Fig.1 Network traffic prediction diagram

Network traffic prediction model plays a very important role in network traffic prediction. Reasonable network traffic prediction model can capture the statistical law of the actual network traffic. On the contrary, unreasonable network traffic prediction model will lead to poor network performance.

\section{FNN Optimized by PLS Pruning Algorithm}

FNN is a kind of multilayer neural network, and when the BP algorithm is used for training, FNN is often called the BP neural network. BP neural networks are usually composed of input layer, hidden layer and output layer. Cybenco has proved that BP neural network with one hidden layer can approach any continual function [7], and the three layer BP neural network is shown in Fig.2.

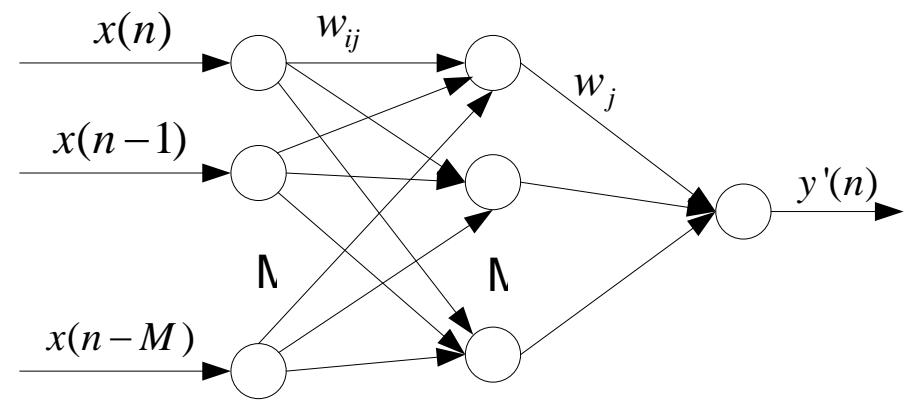

Fig.2 Three layer BP neural network structure

Where $w_{i j}(n)(i=1,2, \mathrm{~L}, M, j=1,2, \mathrm{~L}, N)$ are the weights between the input layer and the hidden layer. $w_{j}(n)(j=1,2, \mathrm{~L}, N)$ are the weights between the hidden layer and the output layer. $x(n)$ is the input data and $y^{\prime}(n)$ is the output data. For network traffic prediction, the network traffic data can be expressed as time series value $x(n)$, the corresponding output is $y^{\prime}(n)$. Let the 
input of the hidden layer is $u_{j}(n)$, and the output of the hidden layer is $v_{j}(n)$, the input of the output layer is $I(n)$, and then the neural network state equation can be expressed as follows

$$
\begin{aligned}
& u_{j}(n)=\sum_{i=1}^{M} w_{i j}(n) x(n-i) \\
& v_{j}(n)=f\left[u_{j}(n)\right] \\
& I(n)=\sum_{j=1}^{N} w_{j}(n) v_{j}(n) \\
& y^{\prime}(n)=f[I(n)]
\end{aligned}
$$

Where $f($.$) is the transfer function of the neural network. This work the sigmoid function is$ choose as the transfer function as follow

$$
f(x)=\frac{1}{1+e^{-x}}
$$

To obtain the robustness training results of the neural network, the observed historical data of network traffic are normalized as follow

$$
x(n)=\frac{x(n)-x_{\min }(n)}{x_{\max }(n)-x_{\min }(n)}
$$

Where $x_{\max }(n)$ and $x_{\min }(n)$ is the maximum and minimum value of the observed historical data of network traffic respectively.

When the training samples and training model are determined, the optimization of the structure of BP neural network can only be carried out for the hidden layer. Here PLS pruning algorithm is used to optimize the hidden layer unit numbers. Let two data matrixes $X$ and $Y$ meet the relationship as follow

$$
Y=X \beta+e
$$

Where $X \in R^{m \times n}$ and $Y \in R^{n \times l}$, $e$ is the noise term. And then the estimation of parameter $\beta$ can be obtained by using the least square method.

$$
\beta=\left(X^{T} X\right)^{-1} X^{T} Y
$$

From Eq.10 can see that, if the data matrix $X$ has strong correlation, and then the parameter estimation must encounter the inverse solution of ill conditioned matrix, which results in big estimation error. PLS algorithm can avoid the inverse solution of ill conditioned matrix by decomposing the matrix $X$ and $Y$ into two internal relations and one external relation follows

$$
\begin{aligned}
& X=T P^{T}+E=\sum_{h=1}^{a} t_{h} p_{h}^{T}+E \\
& Y=U Q^{T}+F=\sum_{h=1}^{a} u_{h} q_{h}^{T}+F \\
& U=T B+R=\sum_{h=1}^{a} b_{h} t_{h}+R
\end{aligned}
$$

Where $T$ and $U$ are the matrixes decomposed by $X$ and $Y . T=\left[t_{1}, t_{2}, \mathrm{~L}, t_{a}\right]$, $U=\left[u_{1}, u_{2}, \mathrm{~L}, u_{a}\right] \cdot t_{h}$ and $u_{h}$ are the primary elements of the matrixes $X$ and $Y, h=1,2, \mathrm{~L}, a$ and $a$ is the number of the number of principal components of the matrix, which can be determined by cross validation method. $P$ and $Q$ are load matrixes, $P=\left[p_{1}, p_{2}, \mathrm{~L}, p_{a}\right]$, $Q=\left[q_{1}, q_{2}, \mathrm{~L}, q_{a}\right] . E, F, R$ are residual matrixes. PLS is a kind of multi variable regression method, which can project high dimensional space information to the low dimension information space which is composed of several independent principal components. PLS solves the problem of multiple correlations in the independent variable, which the information in the independent variable 
and dependent variable is considered when extract the components from the matrixes. In the PLS method, the information of primary components extracted from the independent variables can not only summary the information of the independent variables, but also can has the strongest explanatory of the dependent variables. Therefore, the noise can be removed from the dependent variables and the independent variables. The PLS method can not only eliminate the multi co-linear effects of the original data, but also can deal with the data interfered with noise [8].

The traditional pruning algorithm begins from a sufficiently large neural network, and the hidden layer units are reduced after training convergence. It is a trial method, and the neural network must carry out reconstruction, training and test repeat, thus the computational complexity is considerable large. To reduce the computational complexity, we use the PLS algorithm to optimize the hidden layer units of neural network. The implement process of PLS pruning algorithm can be summarized as follow

Step 1. The collected network traffic data is divided into $m$ groups and each group has the sample data length $l$.

Step 2. Using the $i-t h$ group data as input matrix $X$ and the vector $Y$ as the desired output. The training error threshold is set to $\varepsilon$. When the convergence precision is satisfied $\varepsilon$, carry on the next step.

Step 3. Recording the hidden layer output matrix $G$, the weights $W$ and the output matrix $Y$ at this time. Extracting PLS primary components from the matrix $G$ and $Y, T=\left[t_{1}, t_{2}, \mathrm{~L}, t_{a}\right]$.

Step 4. Computing the correlation coefficients between $t_{i}(1 \leq i \leq a)$ and the output of the hidden layer nodes, and then the hidden layer nodes corresponding to the maximum correlation coefficient are reserved. The number of nodes in the final retention is the number of primary components $a$.

Step 5. Repeating step 2 to choose the next training sample data until $m$ groups data input completely.

Step 6. Deleting redundant nodes in the hidden layer and training the neural network based on the current topological structure and the weights by BP algorithm.

\section{Computer Simulation and Analysis}

In the simulation, the network traffic data is collected from a server of a university campus network. The total number of historical network traffic data is 500 during 10 days, which is shown in Fig.3. The initial network topological structure is $10 \times 40 \times 1$, and the weights are random initialized. The study step size $\mu=0.001$. Using the first 400 data to form a training sample for BP training and the rest of the 100 data is used to test the model. The prediction error is shown in Fig.4. After PLS pruning algorithm, the topology structure of the neural network is optimized to $10 \times 8 \times 1$. Therefore, PLS pruning algorithm can reduce the redundant nodes in the hidden layer. The simulation results show that the proposed method of network traffic prediction reduce the computational complexity and improve the prediction accuracy effectively.

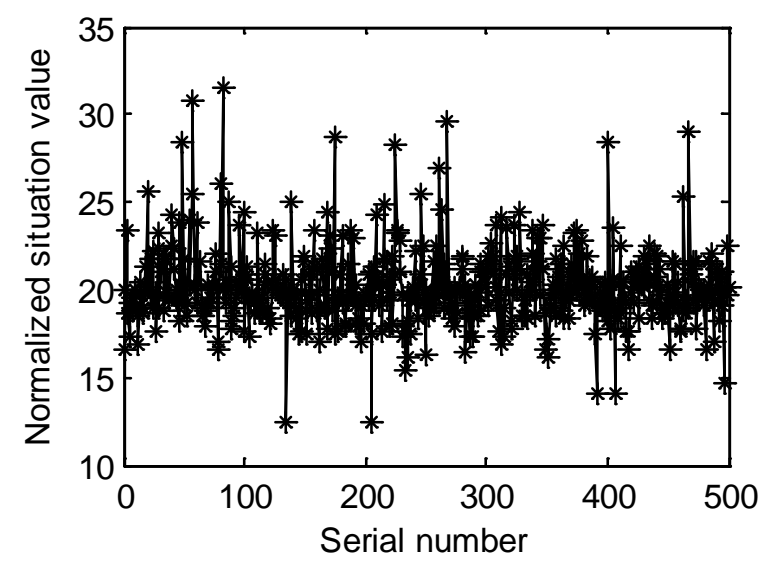

Fig.3 The network traffic historical data

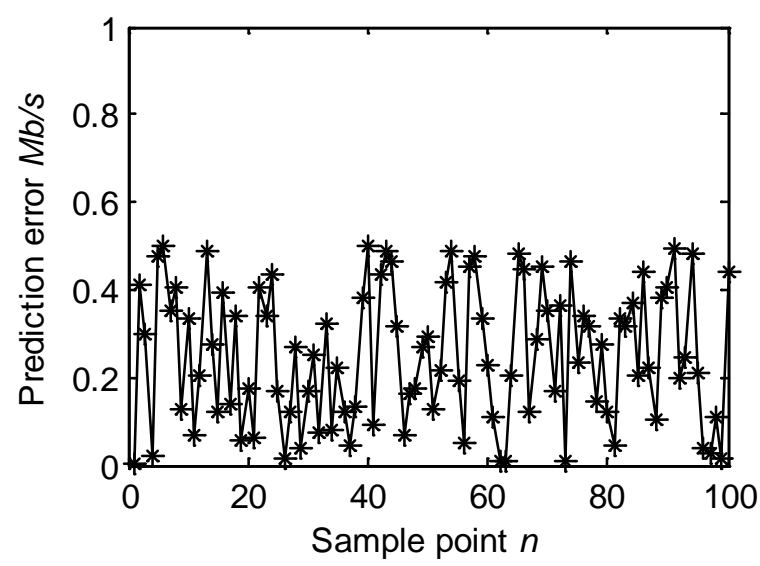

Fig.4 The prediction error 


\section{Conclusion}

This work proposed a network traffic prediction method by FNN with PLS pruning algorithm, which can obtain the ideal topology structure of the neural network. The proposed method can reduce computational complexity of the prediction algorithm and improve the prediction accuracy effectively. The computer simulation result shows the effectiveness of the proposed method.

\section{References}

[1] Z. ZHANG. Network traffic prediction based on combination neural network [J]. Microelectronics \& Computer, 2012, 29(3): 98-101+105.

[2] W. HUANG, Y. HE, H. XIA. Internet traffic prediction based on wavelet neural network [J]. Computer Science, 2011, 38(10A): 296-298+330.

[3] YIN X. D., YANG J., QU C. Q. Prediction research on network traffic of neural network reconstruction based on CPSO algorithm optimization [J]. Computer Engineering and Application, 2014, 50(9): 65-68.

[4] Callado A., Keu R. J., Sadok D., et al. Better network traffic identification through the independent combination of techniques [J]. Journal of Network and Computer Applications, 2010, 33(4): 433-446.

[5] GAO S. T. Chaotic prediction for network traffic flow based on wavelet de-noising and neural network [J]. Computer Engineering and Application, 2012, 48(21): 83-88.

[6] ZHANG F. L., ZHAO Y. L., WANG D., et al. Prediction of network traffic based on traffic characteristic [J]. Computer Science, 2014, 41(4): 86-89+98.

[7] LI H. Y., LI D. Study on network traffic forecasting based on improved neural network [J]. Computer Simulation, 2011, 28(9): 132-135.

[8] Reed R. Pruning algorithm-a survey [J]. IEEE Transactions on Neural Networks, 1993, 4(5): 740-747. 